\title{
A novel role for bioactive lipids in stem cell mobilization during cardiac ischemia:
}

\author{
New Paradigms in Thrombosis: Novel Mediators and Biomarkers \\ Prabhakara R. Nagareddy, PhD, Ahmed Asfour, MD, Yuri M. Klyachkin, PhD, and Ahmed \\ Abdel-Latif, MD, PhD \\ Gill Heart Institute and Division of Cardiovascular Medicine, University of Kentucky, and \\ Lexington VA Medical Center, Lexington, KY, USA
}

\begin{abstract}
Despite major advances in pharmacological and reperfusion therapies, regenerating and/or replacing the infarcted myocardial tissue is an enormous challenge and therefore ischemic heart disease (IHD) remains a major cause of mortality and morbidity worldwide. Adult bone marrow is home for a variety of hematopoietic and non-hematopoietic stem cells including a small subset of primitive cells that carry a promising regenerative potential. It is now well established that myocardial ischemia (MI) induces mobilization of bone marrow-derived cells including differentiated lineage as well as undifferentiated stem cells. While the numbers of stem cells carrying pluripotent features among the mobilized stem cells is small, their regenerative capacity appears immense. Therapies aimed at selective mobilization of these pluripotent stem cells during myocardial ischemia have a promising potential to regenerate the injured myocardium. Emerging evidence suggest that bioactive sphingolipids such as sphingosine-1 phosphate and ceramide-1 phosphate hold a great promise in selective mobilization of pluripotent stem cells to the infarcted region during MI. This review highlights the recent advances in the mechanisms of stem cell mobilization and provides newer evidence in support of bioactive lipids as potential therapeutic agents in the treatment of ischemic heart disease.
\end{abstract}

\section{Keywords}

Stem cell mobilization; myocardial infarction; bioactive lipids; cardiac regeneration

\section{Introduction}

Ischemic heart disease (IHD) is a major cause of mortality and morbidity worldwide and accounts for nearly $20 \%$ of all deaths in United States ${ }^{1}$. The prognosis of acute myocardial infarction (AMI) and ischemic cardiomyopathy (ICM) remains poor despite significant advances in medical therapy and revascularization strategies ${ }^{23}$. Although heart transplantation is the best possible solution to replace the injured myocardium, it is not the

\footnotetext{
Address for correspondence: Ahmed Abdel-Latif, M.D., Ph.D. Division of Cardiovascular Medicine University of Kentucky 740 S. Limestone Street BBSRB Building, Rm 349 Lexington, Kentucky 40536-0200 Tel: (859) 323-0890 Fax: (859) 257-3235 abdellatif@uky.edu.
} 
most viable option because of shortage of organ donors and due to its inherent limitations including graft rejection and continuous use of immunosuppressive drugs. As a result, there are no available treatment strategies that replace the infracted myocardium and the existing therapies are largely palliative.

It has long been assumed that when the heart undergoes ischemic damage, the heart muscle cells do not regenerate and the damage is permanent. This dogma has been challenged in recent years by evidence that heart muscle cells in fact does regenerate. ${ }^{4}$ The first piece of evidence came from a sex mismatched heart transplant study that demonstrated the presence of recipient derived cells in the transplanted heart ${ }^{5,6}$. Since this seminal study, there is a growing line of evidence that cardiomyocytes undergo continuous renewal aided by bone marrow (BM)-derived stem/progenitor cells (SPCs $)^{5-7}$. Further it was demonstrated that the BM-derived cells play a role in the chimerism process of multiple organs including the heart ${ }^{8}$. The mechanisms that promote chimerism appear to be dynamic; responding to significant injury such as myocardial infarction and peaks in the peri-infarct zone 9 . Interestingly, this process is robust enough to achieve the renewal of at least $50 \%$ of all cardiomyocytes in the normal life span ${ }^{10,11}$. Although extensive evidence points to the presence of a dynamic and effective process of cardiomyocyte renewal, the underlying mechanisms are poorly understood.

Acute ischemic myocardial injury initiates multiple repair mechanisms mediated by the mobilization of BMSPCs ${ }^{12-17}$. However, very little is known about the mechanisms that regulate this mobilization. Previous studies in animals have demonstrated that mobilized BMSPCs repopulate the infracted myocardium and can potentially play an important role in the chimerism of cardiomyocytes during usual wear and tear as well as after ischemic injury ${ }^{18}$. On the other hand, the rate of the differentiation of the BMSPCs to cardiomyocytes is low and the majority of the observed benefits can be attributed to paracrine effects ${ }^{19}$. In this review we will discuss the present evidence for the role of the BMSPCs in myocardial regeneration and their viable therapeutic potential. Further, we also provide evidence in support of sphingophospholipids such as sphingosine-1-phosphate (S1P) and ceramide-1phosphate $(\mathrm{C} 1 \mathrm{P})$ as potential therapeutic agents in stem cell therapy.

\section{Phenotypic characteristics of bone marrow-derived pluripotent stem cells}

The bone marrow harbors a heterogeneous pool of hematopoietic and non-hematopoietic stem cell populations. These include the more common lineage-committed cells and the rare pluripotent and embryonic-like stem cells (PSC). The old dogma stating that adults lack pluripotent stem cells has been continuously challenged. In the last decade, multiple studies have demonstrated the presence of PSCs in adult human and animal tissues based on morphology, surface and nuclear marker expression, differentiation capacity and epigenetic characteristics. Based on the expression of Scal (murine stem cell marker) and lack of expression of CD45 (pan-leukocytic marker) and differentiated lineage (Lin) markers, Dr. Ratajczak's group described these PSCs as very small embryonic like stem cells (VSELs) in the murine $\mathrm{BM}^{20,21}$. Subsequently, using corresponding human stem cells markers, VSELs were also isolated from human BM, cord, and peripheral blood ${ }^{22}$. VSELs appear to be morphologically similar to embryonic stem cells with a small diameter compared to more 
committed progenitors and stem cells and a large nucleus containing open-type chromatin surrounded with thin rim of cytoplasm and multiple mitochondria ${ }^{23}$. VSELs also exhibit multiple embryonic and pluripotent surface and nuclear embryonic markers such as Oct 4 , SSEA1/4, Nanog, and Rex1. Further, in vivo and in vitro studies have demonstrated the capability of VSELs to differentiate into multiple cell lines across germ lines including cardiomyocytes $20,21,24$.

The BM also harbors other less defined populations of PSCs such as the mesenchymal stem cells (MSC) ${ }^{25-30}$, multipotent adult progenitor cells (MAPC) ${ }^{31}$, and marrow-isolated multilineage inducible cells (MIAMI) ${ }^{32}$, among others. These populations share the ability to differentiate in vivo and in vitro into various cell lines and sometimes exhibit pluripotent features. It is also possible that different investigators, using diverse methods, may have identified the same or very similar populations and as a result, these populations could have acquired varying nomenclature. However, these stem cell populations may contain, at least in part, VSELs that account for their pluripotent potential.

\section{Mobilization of BM-derived pluripotent stem cells in myocardial ischemia}

BMSPCs including the hematopoietic stem/progenitor cells (HSPCs) are continually released into the peripheral blood $(\mathrm{PB})$ in response to chemotactic gradients and can be detected in the blood under steady state conditions throughout the adult life ${ }^{33}$. A number of factors could contribute to their release into the blood including but not limited to strenuous exercise $^{34}$, tissue or organ injury (including ischemic cardiac events) ${ }^{35,36}$, and administration of pharmacological agents ${ }^{37,38}$. BMSPCs have multi-faceted roles in an adult organism most important of which is lymph-hematopoiesis ${ }^{33}$ and immune surveillance ${ }^{39}$. A continuous chemotactic gradient balance between the BM and the PB may be responsible for their release and homing back to the BM. Among the growing list of chemotactic agents, stromal derived factor-1 (SDF-1) is considered as the major chemotactic factor that influence the destination and homing of BMSPCs ${ }^{40,41}$. Both SDF-1 and its receptor-CXCR4 are highly expressed by osteoblasts and fibroblasts in the BM microenvironment and their interaction results in retention of BMSPCs within the BM niches ${ }^{41}$. It was generally believed that the SDF-1-CXCR4 axis alone was responsible for retention and homing of BMSPCs $^{40,41}$. However, a growing line of evidence describes other major factors that contribute to the mobilization and homing of BMSPCs.

A number of recent studies suggest that other chemotactic factors could also influence the homing/retention of BMSPCs. For example, BMSPCs also express very late antigen-4 (VLA-4, also known as $a_{4} \beta_{1}$-integrin) while the cells in the BM microenvironment express its ligand, vascular adhesion molecule-1 (also known as CD106), further contributing to BMSPCs retention ${ }^{42-44}$. These data suggest that multiple chemotactic mechanisms exist and either independently or in concert with each other influence the ultimate homing or retention of BMSPCs. The growing evidence in support of SDF-1-CXCR4-independent mechanism is also questioning the undisputed exclusive role of SDF-1 as the major mechanism of BMSPC mobilization and homing. For example, recent evidence suggests that the plasma SDF-1 level does not always correlate with mobilization of BMSPCs ${ }^{45-48}$. Similarly, the in vitro chemotaxis assays that demonstrated chemoattractant properties for SDF-1 have, by and 
large, employed supra-physiological concentrations of SDF-1 (100-300 ng/ml) ${ }^{49,50}$, which is about 100 times higher than the SDF-1 concentrations measured in human or murine biological fluids ${ }^{51}$. Another important aspect is that the conditions that induce SDF-1 (e.g. hypoxia) and promote mobilization of BMSPCs (such as G-CSF or a CXCR4 antagonist AMD3100 $)^{44,52-54}$ can also upregulate several proteolytic enzymes in BM cells. Enzymes such as metalloproteinase 2 (MMP-2), MMP-9, cathepsin G and neutrophil elastase can proteolytically cleave and inactivate SDF-1 and CXCR4 resulting in the loss of their chemotactic activity towards BMSPCs ${ }^{55,5652}$. It is important to note that the proteolytic environment would promote HSPC mobilization by decreasing SDF-1-CXCR4-mediated retention (as well as reducing VLA-4-CD106 interaction), but also causes enhanced degradation of SDF-1 thereby impairing its ability to help homing of BMSPCs in target organs ${ }^{44,52,57}$. Taken together, these observations imply the presence of alternative retention and homing mechanisms possibly involving other protease-resistant chemoattractants to make up for the deficiency of the SDF-1 gradient between the BM and PB. These observations led to the exploration of alternative mechanisms including the proteolysisresistant sphingolipids, specifically sphingophospholipids (sphingosine 1-phosphate and ceramide 1-phosphate), which were shown to be potent chemoattractants for BMSPCs.

\section{Sphingophospholipids- Novel lipid mediators as potent stem cell chemo- attractants?}

Sphingolipids are a class of lipids containing a backbone of sphingoid bases, a set of aliphatic amino alcohols that includes sphingosine. They are important structural components of cell membranes. They protect the cell surface against harmful factors by forming a mechanically stable and chemically resistant outer leaflet of the plasma membrane lipid bilayer. Ceramides are $N$-acylated sphingoid bases lacking additional head groups. Ceramide can be deacylated to sphingosine, which is then phosphorylated by sphingosine kinases (SPHK1 or SPHK2) to yield sphingosine 1-phosphate (S1P). Ceramide 1-phosphate (C1P) can be generated by phosphorylation of ceramide ( $\mathrm{N}$-acyl sphingosine) by ceramide kinase ${ }^{58}$. Both S1P and C1P have short half-lives and their plasma and tissue levels are maintained by numerous enzymes. S1P is irreversibly degraded by S1P lyase, and is also regulated by lipid phosphate phosphatases (LPP1-3) and S1P-specific phosphatases (SPP1 and SPP2 $)^{59-63}$, C1P is also regulated by LPP1-3 $3^{59,63}$. The major source of plasma S1P are red blood cells, activated platelets, and extracellular SPHK1 derived from vascular endothelial cells ${ }^{64-66}$, while the major source of plasma C1P comes from intracellular C1P released or leaked from damaged cells ${ }^{67}$.

S1P and C1P interact with a variety of G protein-coupled receptors (GPCR). Five receptor subtypes for $\mathrm{S} 1 \mathrm{P}\left(\mathrm{S}_{1} \mathrm{P}_{1-5}\right)$ have been identified thus far and found widely expressed throughout mammalian tissues. $\mathrm{S}_{1} \mathrm{P}_{1-3}$ are highly expressed throughout the cardiovascular system and also on BMSPCs. The pharmacological actions of $\mathrm{S}_{1} \mathrm{P}_{1}$ are meditated by RasMAP kinase, phosphoinositide (PI) 3-kinase-Akt pathway (PI3K-AKt) and phospholipase $\mathrm{C}$ (PLC) pathways via the inhibitory $\mathrm{G}_{\mathrm{i}}$ protein. $\mathrm{S}_{1} \mathrm{P}_{2}$ and $\mathrm{S}_{1} \mathrm{P}_{3}$ receptor actions are mediated by PLC pathway and Rho pathway via multiple $G$ proteins including $G_{q}, G_{12 / 13}$ and $G_{i}$ subunits ${ }^{68-71}$. The signaling cascade responsible for HSPC migration is by S1P binding to 
either $\mathrm{S}_{1} \mathrm{P}_{1}$ or $\mathrm{S}_{1} \mathrm{P}_{3} 39,72,73$. On the contrary $\mathrm{S}_{1} \mathrm{P}_{2}$ activation by $\mathrm{S} 1 \mathrm{P}$, yields an opposite effect -negatively regulating $\mathrm{HSPC}$ mobilization ${ }^{74} . \mathrm{S}_{1} \mathrm{P}_{4}$ and $\mathrm{S}_{1} \mathrm{P}_{5}$ receptors play a role in the immune and nervous system, respectively. While the receptor for C1P is yet to be identified, it's signaling is sensitive to pertussis toxin, thereby implicating a $G_{i}$ protein coupled receptor ${ }^{75,76}$.

\section{Sphingosine 1-phosphate is a potent BMSPCs chemoattractant?}

With the discovery of S1P receptors on BMSPCs and subsequent characterization as GPCR, it was hypothesized that S1P might possess biological roles similar to other chemokines. This led to the identification of S1P as a chemotactic agent by Seitz et al ${ }^{45}$. Further, it was demonstrated that the gradient of S1P between BM and PB determines HSPCs egress ${ }^{47}$. While SDF-1 still has a significant role in retention of HSPCs in BM niches, it might not be as crucial in the mobilization and homing of BMSPCs including HSPCs ${ }^{47}$. For example, plasma derived from normal and mobilized peripheral blood strongly attracts murine BM HSPCs independent of plasma SDF-1 levels. On the contrary when lipids (including S1P) were removed from the plasma by charcoal stripping, it abolished HSPCs chemotaxis but did not affect responsiveness towards SDF- ${ }^{47}$. It was further demonstrated that already steady state S1P plasma levels create a gradient favoring HSPCs egress from the BM.

As previously described, HSPCs are retained in the BM by SDF-1-CXCR4 and VLA4VCAM1 interactions. Disruption of these interactions either by a CXCR4 antagonist (AMD3100) or triggering a proteolytic environment in the BM would release HSPCs form their niches or therefore allow them to follow the bioactive lipids gradient to PB. G-CSF, a frequently used mobilizing agent also mobilizes BMSPCs by increasing plasma S1P levels ${ }^{77}$. G-CSF triggers complement complex activation resulting in the stimulation of granulocytes and subsequent release of proteolytic enzymes in the BM microenvironment thus disrupting the interaction between stem cells and osteoclasts in the BM niches ${ }^{53}$. Interestingly, the lasting effect of G CSF promotes complement cascade (CC) activation and formation of the membrane attack complex (MAC) that was shown to interact with erythrocytes and could contribute to the release of bioactive lipids in the plasma ${ }^{78}$. While erythrocytes serve as the major reservoir of S1P in the $\mathrm{PB}^{46,79}$, they are highly protected from MAC by CD59 and decay-accelerating factor (DAF) receptors ${ }^{80}$. However, it was subsequently demonstrated that expression of these receptors on erythrocytes does not give complete protection from activated MAC since G-CSF-induced MAC exposure resulted in increase in plasma S1P levels promoting HSPCs egress ${ }^{47}$.

While it has been established that S1P plays a significant role in HSPC trafficking, the mechanisms that mediate this remains unclear. Recent evidence suggests that SDF-1 and S1P may work synergistically to facilitate egress of HSPCs out of the $\mathrm{BM}^{81}$. It was also

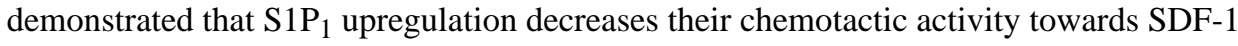
due to reduced cell surface expression of CXCR4 suggesting a potential interaction and feedback mechanisms between S1P and SDF-1 ${ }^{82}$. These observations were recently confirmed by Golan et al who showed that short-term inhibition of $\mathrm{S} 1 \mathrm{P} / \mathrm{S} 1 \mathrm{P}_{1}$ axis during steady state conditions or during CXCR4 inhibition (via AMD3100 administration) reduces SDF-1 in the plasma ${ }^{83}$. Interestingly, generation of reactive oxygen species (ROS) via $\mathrm{S}_{1} \mathrm{P}_{1}$ 
signaling was also implicated in HSPC mobilization through the release of SDF- ${ }^{84}$. Since previous studies showed that ROS inhibition reduces SDF-1 secretion during AMD3100induced mobilization ${ }^{85}$ it was thought that ROS signaling might also contribute to SDF-1 secretion. Indeed it was confirmed that ROS signaling induced SDF-1 secretion thereby facilitating HSPCs egress ${ }^{83}$.

The interaction of S1P-SDF-1 in prompting HSPC egress was further demonstrated with the help of FTY720, a potent $\mathrm{S}_{1} \mathrm{P}_{1}$ desensitizing agent which causes S1P receptor internalization ${ }^{86}$. Interestingly, administration of FTY720 for 24 hours resulted in increased plasma SDF-1 levels but had no effect on HSPC egress. FTY720 treatment did reduce BM ROS signaling, due to $\mathrm{S}_{1} \mathrm{P}_{1}$ down regulation, again pointing out the requirement of $\mathrm{S}_{1} \mathrm{P}_{1}$ signaling in HSPC egress. Furthermore, mice that were treated with BM-specific S1P lyase inhibitor 4-deoxypyrodixne (DOP) that resulted in increase of S1P level in BM microenvironment and lymphatic organs, were poor mobilizers of HSPCs, and showed defective egress of lymphocytes from lymphatic organs. This could be explained by increase in BM ROS levels ${ }^{83}$. Together these observations suggest that the increased concentrations of S1P and SDF-1 in the BM negatively affect HSPC egress, further highlighting the fact that both S1P and SDF-1 levels must be tightly regulated for balanced HSPCs mobilization.

\section{The role of bioactive lipids and the innate immunity in stem cell mobilization and homing during myocardial ischemia}

While bioactive lipids such as S1P and C1P are powerful mobilizers of BMSPCs, their role in BMSPCs' mobilization and homing to ischemic myocardium is not well understood. The role of other chemoattractants in BMSPCs homing to a hostile environment such as the infarcted myocardium is also unclear. We recently examined the role of bioactive lipids, elements of complement cascade, and anti-microbial peptides in BMSPC mobilization and homing during $\mathrm{MI}^{87}$. Our data shows elevated level of S1P and C1P in the plasma of MI patients shortly after the onset of injury. Increased S1P and C1P levels were correlated to elevated numbers of circulating BMSPCs suggesting a role of bioactive lipids in BMSPCs mobilization post-MI. Our speculations were corroborated by a modified Boyden chamber assay (chemotaxis assay) where we observed increased BMSPC chemotaxis towards plasma obtained from patients at peak BMSPCs mobilization. Moreover, this migration was selectively blocked by W146, a specific $\mathrm{S}_{1} \mathrm{P}_{1}$ antagonist, further implicating $\mathrm{S} 1 \mathrm{P}$ as a potent BMSPCs chemoattractant during MI. As previously described, MI induces a potent proteolytic environment in which numerous enzymes such as MMPs and proteases irreversibly degrade potent BMSPCs chemoattractants such as SDF-1.

Recent evidence suggests a pivotal role for antimicrobial protein cathelicidin such as LL-37 in humans in sensitizing BMSPCs towards significantly lower levels of SDF-1 ${ }^{88}$. Our data suggests that cathelicidins are secreted by cardiac fibroblasts in response to hypoxia and can compensate for the reduced level of SDF-1 in this proteolytic environment and aid in BMSPC homing to the injured myocardium ${ }^{87}$. Similar effect has been observed in presence of cleavage fragment of third complement protein component - C3a anaphylatoxin ${ }^{89-91}$. Both LL-37 and C3a sensitize BMSPCs by incorporating CXCR4 into the lipid rafts thereby augmenting CXCR4 signaling. We observed similar priming effect with LL37 and C3a in 
enhancing the migration of peripheral blood cells isolated from MI patients towards low, yet physiological, levels of SDF-1. Taken together our findings highlight the importance of bioactive lipids (S1P, C1P) and elements of innate immunity (e.g., MAC, C3a, and LL-37) in the mobilization and homing of BMSPCs to the ischemic myocardium.

\section{Conclusions}

Ischemic heart disease is approaching epidemic levels in the United States and the Western world. Unfortunately, available therapies are largely symptomatic with no strategies to repair the damaged myocardium, which has initiated increasing interest in regenerative therapies particularly those utilizing bone marrow derived stem cells. However, studies examining the therapeutic utility of BMSPC infusion ${ }^{92}$ or their mobilization ${ }^{93}$ after MI achieved limited success due to the lack of focused and mechanism-based approaches. Multiple new therapies that modulate plasma levels of S1P or its receptors' expression are approved by the FDA and can be utilized in improving the mobilization of BMSPCs in myocardial ischemia in future myocardial regenerative studies (Figure 2). Similarly, priming BM-derived cells with LL-37 can be used to improve their homing to the ischemic myocardium and thus overcome a major hurdle in stem cell regenerative myocardial therapies. We are currently examining both strategies in our laboratory to improve the mobilization and homing of BMSPCs to the ischemic myocardium. Most importantly, the success of stem cell therapy depends on the timing, dosing and the route of administration. The temporal window of opportunity for successful mobilization of BMSCs is bound by the heightened inflammatory response in the first 4 days post MI and the formation of scar as soon as 10-14 post-MI. Therefore, development of successful therapies aimed at selective mobilization of BMSCs depends on timing and restricting inflammatory cell egress. One such strategy could be the suppression of CCR 2 in pro-inflammatory monocytes ${ }^{94}$, a major monocyte subset that is recruited to injured myocardium ${ }^{95}$ at the same time BMSPCs are also recruited. Nonetheless, further research is clearly required in the optimization of timing protocols and the method and route of delivery of BMSCs.

\section{References}

1. Rosamond W, et al. Heart disease and stroke statistics--2008 update: a report from the American Heart Association Statistics Committee and Stroke Statistics Subcommittee. Circulation. 2008; 117:e25-146. [PubMed: 18086926]

2. Braunwald E, Bristow MR. Congestive heart failure: Fifty years of progress. Circulation. 2000; 102:14-23. [PubMed: 10880409]

3. McMurray JJ, Pfeffer MA. Heart failure. Lancet. 2005; 365:1877-1889. [PubMed: 15924986]

4. Orlic D, et al. Bone marrow cells regenerate infarcted myocardium. Nature. 2001; 410:701-705. [PubMed: 11287958]

5. Rupp S, et al. Characterization of long-term endogenous cardiac repair in children after heart transplantation. European heart journal. 2008; 29:1867-1872. [PubMed: 18511408]

6. Quaini F, et al. Chimerism of the transplanted heart. The New England journal of medicine. 2002; 346:5-15. [PubMed: 11777997]

7. Deb A, et al. Bone marrow-derived cardiomyocytes are present in adult human heart: A study of gender-mismatched bone marrow transplantation patients. Circulation. 2003; 107:1247-1249. [PubMed: 12628942] 
8. de Weger RA, et al. Stem cell-derived cardiomyocytes after bone marrow and heart transplantation. Bone Marrow Transplant. 2008; 41:563-569. [PubMed: 18037937]

9. Hsieh PC, et al. Evidence from a genetic fate-mapping study that stem cells refresh adult mammalian cardiomyocytes after injury. Nature medicine. 2007; 13:970-974.

10. Kajstura J, et al. Cardiomyogenesis in the aging and failing human heart. Circulation. 2012; 126:1869-1881. [PubMed: 22955965]

11. Bergmann O, et al. Evidence for cardiomyocyte renewal in humans. Science. 2009; 324:98-102. [PubMed: 19342590]

12. Massa M, et al. Increased circulating hematopoietic and endothelial progenitor cells in the early phase of acute myocardial infarction. Blood. 2005; 105:199-206. [PubMed: 15345590]

13. Lev EI, et al. Circulating endothelial progenitor cells and coronary collaterals in patients with nonST segment elevation myocardial infarction. Journal of vascular research. 2005; 42:408-414. [PubMed: 16088214]

14. Shintani S, et al. Mobilization of endothelial progenitor cells in patients with acute myocardial infarction. Circulation. 2001; 103:2776-2779. [PubMed: 11401930]

15. Wojakowski W, et al. Mobilization of bone marrow-derived Oct-4+ SSEA-4+ very small embryonic-like stem cells in patients with acute myocardial infarction. Journal of the American College of Cardiology. 2009; 53:1-9. [PubMed: 19118716]

16. Wojakowski W, et al. Mobilization of CD34(+), CD117(+), CXCR4(+), c-met(+) stem cells is correlated with left ventricular ejection fraction and plasma NT-proBNP levels in patients with acute myocardial infarction. European heart journal. 2006; 27:283-289. [PubMed: 16267071]

17. Abdel-Latif A, et al. Evidence of mobilization of pluripotent stem cells into peripheral blood of patients with myocardial ischemia. Experimental hematology. 2010; 38:1131-1142. e1131. [PubMed: 20800644]

18. Fukuhara S, Tomita S, Nakatani T, Yutani C, Kitamura S. Endogenous bone-marrow-derived stem cells contribute only a small proportion of regenerated myocardium in the acute infarction model. The Journal of heart and lung transplantation : the official publication of the International Society for Heart Transplantation. 2005; 24:67-72.

19. Loffredo FS, Steinhauser ML, Gannon J, Lee RT. Bone marrow-derived cell therapy stimulates endogenous cardiomyocyte progenitors and promotes cardiac repair. Cell Stem Cell. 2011; 8:389398. [PubMed: 21474103]

20. Kucia M, et al. Morphological and molecular characterization of novel population of CXCR4+ SSEA-4+ Oct-4+ very small embryonic-like cells purified from human cord blood: preliminary report. Leukemia : official journal of the Leukemia Society of America, Leukemia Research Fund, U.K. 2007; 21:297-303.

21. Kucia M, et al. A population of very small embryonic-like (VSEL) CXCR4(+)SSEA-1(+)Oct-4+ stem cells identified in adult bone marrow. Leukemia. 2006; 20:857-869. [PubMed: 16498386]

22. Liu R, Klich I, Ratajczak J, Ratajczak MZ, Zuba-Surma EK. Erythrocyte-derived microvesicles may transfer phosphatidylserine to the surface of nucleated cells and falsely 'mark' them as apoptotic. European journal of haematology. 2009; 83:220-229. [PubMed: 19456851]

23. Zuba-Surma EK, et al. Morphological characterization of very small embryonic-like stem cells (VSELs) by ImageStream system analysis. J Cell Mol Med. 2008; 12:292-303. [PubMed: 18031297]

24. Kucia MJ, et al. Evidence that very small embryonic-like stem cells are mobilized into peripheral blood. Stem Cells. 2008; 26:2083-2092. [PubMed: 18511604]

25. Hattan N, et al. Purified cardiomyocytes from bone marrow mesenchymal stem cells produce stable intracardiac grafts in mice. Cardiovasc Res. 2005; 65:293-295. [PubMed: 15639465]

26. Kawada $\mathrm{H}$, et al. Nonhematopoietic mesenchymal stem cells can be mobilized and differentiate into cardiomyocytes after myocardial infarction. Blood. 2004; 104:3581-3587. [PubMed: 15297308]

27. Makino S, et al. Cardiomyocytes can be generated from marrow stromal cells in vitro. J Clin Invest. 1999; 103:697-705. [PubMed: 10074487]

28. Shake J, et al. Mesenchymal stem cell implantation in a swine myocardial infarct model: engraftment and functional effects. Ann Thorac Surg. 2002; 73:1919-1925. [PubMed: 12078791] 
29. Oswald J, et al. Mesenchymal stem cells can be differentiated into endothelial cells in vitro. Stem Cells. 2004; 22:377-384. [PubMed: 15153614]

30. Silva G, et al. Mesenchymal stem cells differentiate into an endothelial phenotype, enhance vascular density, and improve heart function in a canine chronic ischemia model. Circulation. 2005; 111:150-156. [PubMed: 15642764]

31. Jiang Y, et al. Pluripotency of mesenchymal stem cells derived from adult marrow. Nature. 2002; 418:41-49. [PubMed: 12077603]

32. D'Ippolito G, et al. Marrow-isolated adult multilineage inducible (MIAMI) cells, a unique population of postnatal young and old human cells with extensive expansion and differentiation potential. J Cell Sci. 2004; 117:2971-2981. [PubMed: 15173316]

33. Bonig H, Priestley GV, Oehler V, Papayannopoulou T. Hematopoietic progenitor cells (HPC) from mobilized peripheral blood display enhanced migration and marrow homing compared to steadystate bone marrow HPC. Exp Hematol. 2007; 35:326-334. [PubMed: 17258081]

34. Mobius-Winkler S, et al. Time-dependent mobilization of circulating progenitor cells during strenuous exercise in healthy individuals. J Appl Physiol. 2009; 107:1943-1950. [PubMed: 19797690]

35. Wojakowski W, Tendera M. Mobilization of bone marrow-derived progenitor cells in acute coronary syndromes. Folia Histochem Cytobiol. 2005; 43:229-232. [PubMed: 16382890]

36. Wojakowski W, Landmesser U, Bachowski R, Jadczyk T, Tendera M. Mobilization of stem and progenitor cells in cardiovascular diseases. Leukemia. 2012; 26:23-33. [PubMed: 21788948]

37. Paczkowska E, et al. Clinical evidence that very small embryonic-like stem cells are mobilized into peripheral blood in patients after stroke. Stroke. 2009; 40:1237-1244. [PubMed: 19246697]

38. Lee HM, et al. Mobilization studies in complement-deficient mice reveal that optimal AMD3100 mobilization of hematopoietic stem cells depends on complement cascade activation by AMD3100-stimulated granulocytes. Leukemia. 2010; 24:573-582. [PubMed: 20033053]

39. Massberg S, et al. Immunosurveillance by hematopoietic progenitor cells trafficking through blood, lymph, and peripheral tissues. Cell. 2007; 131:994-1008. [PubMed: 18045540]

40. Wright DE, Bowman EP, Wagers AJ, Butcher EC, Weissman IL. Hematopoietic stem cells are uniquely selective in their migratory response to chemokines. J Exp Med. 2002; 195:1145-1154. [PubMed: 11994419]

41. Lapidot T, Dar A, Kollet O. How do stem cells find their way home? Blood. 2005; 106:19011910. [PubMed: 15890683]

42. Peled A, et al. The chemokine SDF-1 stimulates integrin-mediated arrest of CD34(+) cells on vascular endothelium under shear flow. J Clin Invest. 1999; 104:1199-1211. [PubMed: 10545519]

43. Levesque JP, Takamatsu Y, Nilsson SK, Haylock DN, Simmons PJ. Vascular cell adhesion molecule-1 (CD106) is cleaved by neutrophil proteases in the bone marrow following hematopoietic progenitor cell mobilization by granulocyte colony-stimulating factor. Blood. 2001; 98:1289-1297. [PubMed: 11520773]

44. Levesque JP, Hendy J, Takamatsu Y, Simmons PJ, Bendall LJ. Disruption of the CXCR4/CXCL12 chemotactic interaction during hematopoietic stem cell mobilization induced by GCSF or cyclophosphamide. J Clin Invest. 2003; 111:187-196. [PubMed: 12531874]

45. Seitz G, Boehmler AM, Kanz L, Mohle R. The role of sphingosine 1-phosphate receptors in the trafficking of hematopoietic progenitor cells. Ann N Y Acad Sci. 2005; 1044:84-89. [PubMed: 15958700]

46. Hanel P, Andreani P, Graler MH. Erythrocytes store and release sphingosine 1-phosphate in blood. FASEB J. 2007; 21:1202-1209. [PubMed: 17215483]

47. Ratajczak MZ, et al. Novel insight into stem cell mobilization-plasma sphingosine-1-phosphate is a major chemoattractant that directs the egress of hematopoietic stem progenitor cells from the bone marrow and its level in peripheral blood increases during mobilization due to activation of complement cascade/membrane attack complex. Leukemia. 2010; 24:976-985. [PubMed: 20357827]

48. Marquez-Curtis LA, Turner AR, Sridharan S, Ratajczak MZ, Janowska-Wieczorek A. The ins and outs of hematopoietic stem cells: studies to improve transplantation outcomes. Stem Cell Rev. 2011; 7:590-607. [PubMed: 21140298] 
49. Christopherson KW 2nd, Hangoc G, Mantel CR, Broxmeyer HE. Modulation of hematopoietic stem cell homing and engraftment by CD26. Science. 2004; 305:1000-1003. [PubMed: 15310902]

50. Basu S, Ray NT, Atkinson SJ, Broxmeyer HE. Protein phosphatase 2A plays an important role in stromal cell-derived factor-1/CXC chemokine ligand 12-mediated migration and adhesion of CD34+ cells. J Immunol. 2007; 179:3075-3085. [PubMed: 17709522]

51. Gazitt Y, Liu Q. Plasma levels of SDF-1 and expression of SDF-1 receptor on CD34+ cells in mobilized peripheral blood of non-Hodgkin's lymphoma patients. Stem Cells. 2001; 19:37-45. [PubMed: 11209089]

52. Kim $\mathrm{CH}$, et al. Conditioning for hematopoietic transplantation activates the complement cascade and induces a proteolytic environment in bone marrow: a novel role for bioactive lipids and soluble C5b-C9 as homing factors. Leukemia. 2012; 26:106-116. [PubMed: 21769103]

53. Greenbaum AM, Link DC. Mechanisms of G-CSF-mediated hematopoietic stem and progenitor mobilization. Leukemia. 2011; 25:211-217. [PubMed: 21079612]

54. Pusic I, DiPersio JF. Update on clinical experience with AMD3100, an SDF-1/CXCL12-CXCR4 inhibitor, in mobilization of hematopoietic stem and progenitor cells. Curr Opin Hematol. 2010; 17:319-326. [PubMed: 20473162]

55. McQuibban GA, et al. Matrix metalloproteinase activity inactivates the CXC chemokine stromal cell-derived factor-1. The Journal of biological chemistry. 2001; 276:43503-43508. [PubMed: 11571304]

56. McQuibban GA, et al. Matrix metalloproteinase processing of monocyte chemoattractant proteins generates CC chemokine receptor antagonists with anti-inflammatory properties in vivo. Blood. 2002; 100:1160-1167. [PubMed: 12149192]

57. Pelus LM, Bian H, King AG, Fukuda S. Neutrophil-derived MMP-9 mediates synergistic mobilization of hematopoietic stem and progenitor cells by the combination of G-CSF and the chemokines GRObeta/CXCL2 and GRObetaT/CXCL2delta4. Blood. 2004; 103:110-119. [PubMed: 12958067]

58. Boath A, et al. Regulation and traffic of ceramide 1-phosphate produced by ceramide kinase: comparative analysis to glucosylceramide and sphingomyelin. J Biol Chem. 2008; 283:85178526. [PubMed: 18086664]

59. Sciorra VA, Morris AJ. Roles for lipid phosphate phosphatases in regulation of cellular signaling. Biochim Biophys Acta. 2002; 1582:45-51. [PubMed: 12069809]

60. Long J, et al. Regulation of cell survival by lipid phosphate phosphatases involves the modulation of intracellular phosphatidic acid and sphingosine 1-phosphate pools. Biochem J. 2005; 391:2532. [PubMed: 15960610]

61. Mechtcheriakova D, et al. Sphingosine 1-phosphate phosphatase 2 is induced during inflammatory responses. Cell Signal. 2007; 19:748-760. [PubMed: 17113265]

62. Schwab SR, et al. Lymphocyte sequestration through S1P lyase inhibition and disruption of S1P gradients. Science. 2005; 309:1735-1739. [PubMed: 16151014]

63. Brindley DN, English D, Pilquil C, Buri K, Ling ZC. Lipid phosphate phosphatases regulate signal transduction through glycerolipids and sphingolipids. Biochim Biophys Acta. 2002; 1582:33-44. [PubMed: 12069808]

64. Yatomi Y, Ozaki Y, Ohmori T, Igarashi Y. Sphingosine 1-phosphate: synthesis and release. Prostaglandins. 2001; 64:107-122. [PubMed: 11331099]

65. Kihara A, Igarashi Y. Production and release of sphingosine 1-phosphate and the phosphorylated form of the immunomodulator FTY720. Biochim Biophys Acta. 2008; 1781:496-502. [PubMed: 18555808]

66. Venkataraman K, et al. Extracellular export of sphingosine kinase-1a contributes to the vascular S1P gradient. Biochem J. 2006; 397:461-471. [PubMed: 16623665]

67. Lamour NF, et al. Ceramide kinase uses ceramide provided by ceramide transport protein: localization to organelles of eicosanoid synthesis. J Lipid Res. 2007; 48:1293-1304. [PubMed: 17392267]

68. Takuwa Y. Subtype-specific differential regulation of Rho family $\mathrm{G}$ proteins and cell migration by the Edg family sphingosine-1-phosphate receptors. Biochim Biophys Acta. 2002; 1582:112-120. [PubMed: 12069818] 
69. Spiegel S, Milstien S. Sphingosine-1-phosphate: an enigmatic signalling lipid. Nat Rev Mol Cell Biol. 2003; 4:397-407. [PubMed: 12728273]

70. Ishii I, Fukushima N, Ye X, Chun J. Lysophospholipid receptors: signaling and biology. Annu Rev Biochem. 2004; 73:321-354. [PubMed: 15189145]

71. Hla T. Physiological and pathological actions of sphingosine 1-phosphate. Semin Cell Dev Biol. 2004; 15:513-520. [PubMed: 15271296]

72. Jo E, et al. S1P1-selective in vivo-active agonists from high-throughput screening: off-the-shelf chemical probes of receptor interactions, signaling, and fate. Chem Biol. 2005; 12:703-715. [PubMed: 15975516]

73. Walter DH, et al. Sphingosine-1-phosphate stimulates the functional capacity of progenitor cells by activation of the CXCR4-dependent signaling pathway via the S1P3 receptor. Arterioscler Thromb Vasc Biol. 2007; 27:275-282. [PubMed: 17158356]

74. Michaud J, Im DS, Hla T. Inhibitory role of sphingosine 1-phosphate receptor 2 in macrophage recruitment during inflammation. J Immunol. 2010; 184:1475-1483. [PubMed: 20042570]

75. Gomez-Munoz A, Kong JY, Salh B, Steinbrecher UP. Ceramide-1-phosphate blocks apoptosis through inhibition of acid sphingomyelinase in macrophages. J Lipid Res. 2004; 45:99-105. [PubMed: 14523050]

76. Granado MH, et al. Ceramide 1-phosphate (C1P) promotes cell migration Involvement of a specific C1P receptor. Cell Signal. 2009; 21:405-412. [PubMed: 19041940]

77. Ryan MA, et al. Pharmacological inhibition of EGFR signaling enhances G-CSF-induced hematopoietic stem cell mobilization. Nat Med. 2010; 16:1141-1146. [PubMed: 20871610]

78. Lindorfer MA, et al. A novel approach to preventing the hemolysis of paroxysmal nocturnal hemoglobinuria: both complement-mediated cytolysis and $\mathrm{C} 3$ deposition are blocked by a monoclonal antibody specific for the alternative pathway of complement. Blood. 2010; 115:22832291. [PubMed: 20068220]

79. Ohkawa R, et al. Plasma sphingosine-1-phosphate measurement in healthy subjects: close correlation with red blood cell parameters. Ann Clin Biochem. 2008; 45:356-363. [PubMed: 18583619]

80. Bessler M, Hiken J. The pathophysiology of disease in patients with paroxysmal nocturnal hemoglobinuria. Hematology Am Soc Hematol Educ Program. 2008:104-110. [PubMed: 19074066]

81. Whetton AD, Lu Y, Pierce A, Carney L, Spooncer E. Lysophospholipids synergistically promote primitive hematopoietic cell chemotaxis via a mechanism involving Vav 1. Blood. 2003; 102:2798-2802. [PubMed: 12829605]

82. Ryser MF, Ugarte F, Lehmann R, Bornhauser M, Brenner S. S1P(1) overexpression stimulates S1P-dependent chemotaxis of human CD34+ hematopoietic progenitor cells but strongly inhibits SDF-1/CXCR4-dependent migration and in vivo homing. Mol Immunol. 2008; 46:166-171. [PubMed: 18760838]

83. Golan K, et al. S1P promotes murine progenitor cell egress and mobilization via S1P1-mediated ROS signaling and SDF-1 release. Blood. 2012; 119:2478-2488. [PubMed: 22279055]

84. Tesio M, et al. Enhanced c-Met activity promotes G-CSF-induced mobilization of hematopoietic progenitor cells via ROS signaling. Blood. 2011; 117:419-428. [PubMed: 20585044]

85. Dar A, et al. Rapid mobilization of hematopoietic progenitors by AMD3100 and catecholamines is mediated by CXCR4-dependent SDF-1 release from bone marrow stromal cells. Leukemia. 2011; 25:1286-1296. [PubMed: 21494253]

86. Brinkmann V. FTY720: mechanism of action and potential benefit in organ transplantation. Yonsei Med J. 2004; 45:991-997. [PubMed: 15627289]

87. Karapetyan AV, et al. Bioactive lipids and cationic antimicrobial peptides as new potential regulators for trafficking of bone marrow-derived stem cells in patients with acute myocardial infarction. Stem Cells Dev. 2013; 22:1645-1656. [PubMed: 23282236]

88 . $\mathrm{Wu} \mathrm{W}$, et al. The bone marrow-expressed antimicrobial cationic peptide LL-37 enhances the responsiveness of hematopoietic stem progenitor cells to an SDF-1 gradient and accelerates their engraftment after transplantation. Leukemia : official journal of the Leukemia Society of America, Leukemia Research Fund, U.K. 2011 
89. Wysoczynski M, et al. Defective engraftment of C3aR-/- hematopoietic stem progenitor cells shows a novel role of the C3a-C3aR axis in bone marrow homing. Leukemia. 2009; 23:14551461. [PubMed: 19357704]

90. Ratajczak J, et al. Mobilization studies in mice deficient in either $\mathrm{C} 3$ or C3a receptor $(\mathrm{C} 3 \mathrm{aR})$ reveal a novel role for complement in retention of hematopoietic stem/progenitor cells in bone marrow. Blood. 2004; 103:2071-2078. [PubMed: 14604969]

91. Reca R, et al. Functional receptor for $\mathrm{C} 3 \mathrm{a}$ anaphylatoxin is expressed by normal hematopoietic stem/progenitor cells, and C3a enhances their homing-related responses to SDF-1. Blood. 2003; 101:3784-3793. [PubMed: 12511407]

92. Abdel-Latif A, et al. Adult bone marrow-derived cells for cardiac repair: a systematic review and meta-analysis. Arch Intern Med. 2007; 167:989-997. [PubMed: 17533201]

93. Abdel-Latif A, et al. Granulocyte colony-stimulating factor therapy for cardiac repair after acute myocardial infarction: a systematic review and meta-analysis of randomized controlled trials. Am Heart J. 2008; 156:216-226. e219. [PubMed: 18657649]

94. Leuschner F, et al. Therapeutic siRNA silencing in inflammatory monocytes in mice. Nature biotechnology. 2011; 29:1005-1010.

95. Nahrendorf M, et al. The healing myocardium sequentially mobilizes two monocyte subsets with divergent and complementary functions. J Exp Med. 2007; 204:3037-3047. [PubMed: 18025128] 


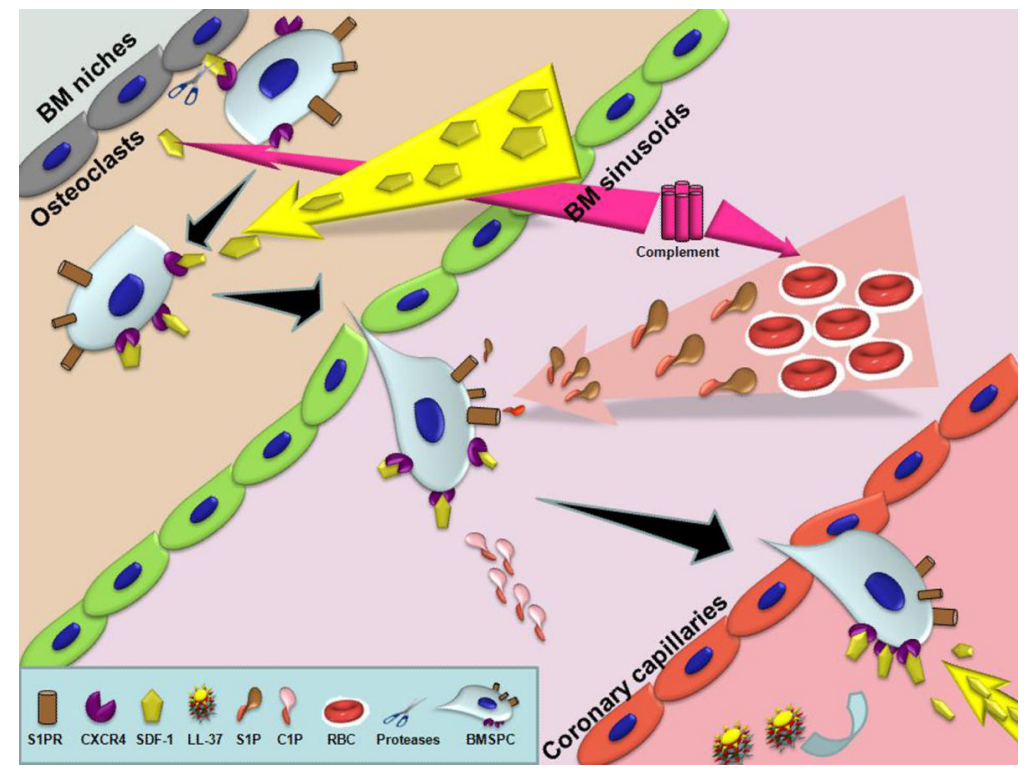

Figure 1. Sequence of events in BMSPC mobilization from the BM towards ischemic myocardium during MI

Acute myocardial infarction initiates an inflammatory response resulting in the activation of the complement cascade that in turn releases proteases (by granulocytes and osteoclasts) in the BM, which proteolytically inactivate the SDF-1-CXCR4 interaction between BM osteoclasts and BMSPCS. The now mobilized BMSPCs follow an increasing SDF-1 and bioactive lipid (S1P and C1P) gradient to exit the BM niches into the PB. Acute inflammation also promotes release of cathelicidins (LL-37), which facilitate clustering of CXCR4 into lipid rafts thereby increasing their sensitivity towards lower levels of local SDF-1 in the injured myocardium. Together, the increased sensitivity towards SDF-1 and bioactive lipid gradients facilitate BMSPC homing towards ischemic myocardium. 


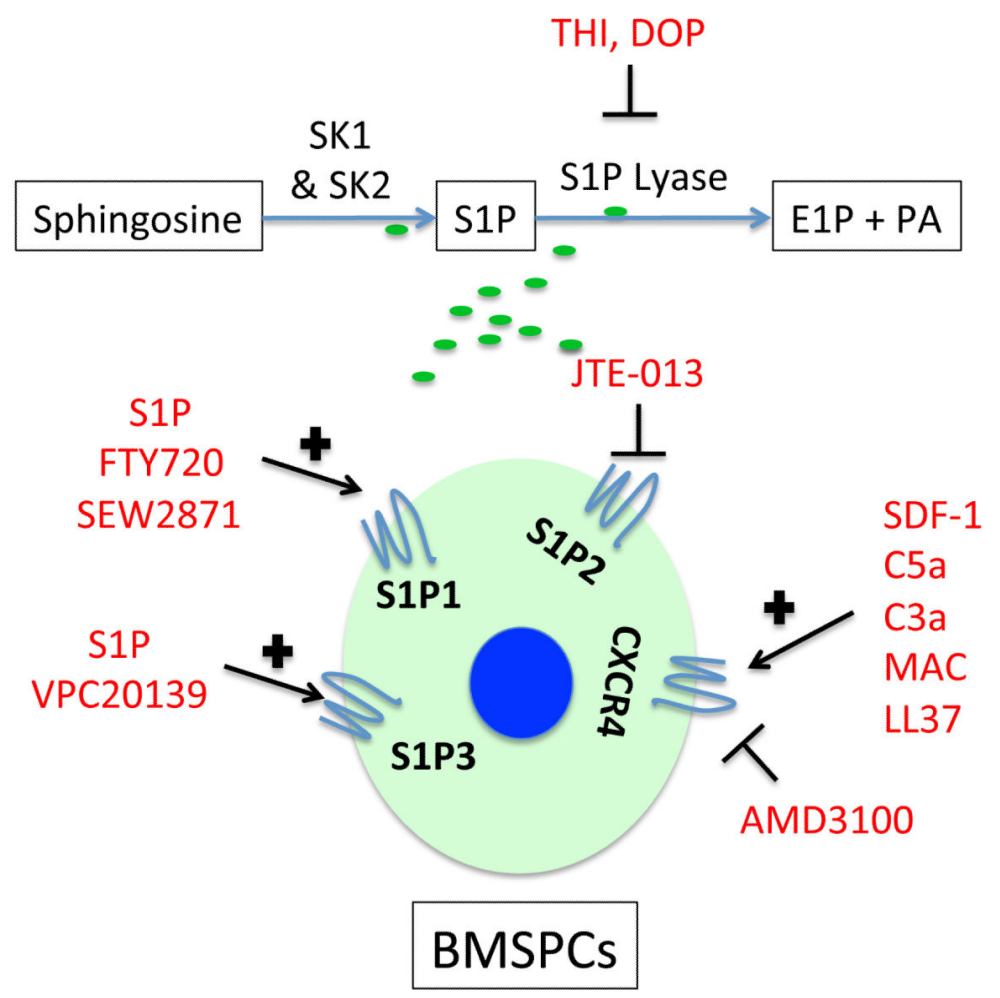

Figure 2. Potential strategies to improve homing of HSPCs by targeting the S1P-S1P receptor axis

S1P-mediated mobilization and homing of BMSPCs is mediated by $\mathrm{S}_{1} \mathrm{P}_{1}$ and $\mathrm{S}_{\mathrm{P}} \mathrm{P}_{3}$ while $\mathrm{S}_{1} \mathrm{P}_{2}$ is known to inhibit this mobilization. Mobilization of BMSPCs could be improved by upregulating S1P levels via inhibition of sphingosine-1 lyase enzyme, using DOP or THI, thus creating a gradient for stem cell mobilization. Further, specific agonists of $\mathrm{S}_{1} \mathrm{P}_{1}$ (such

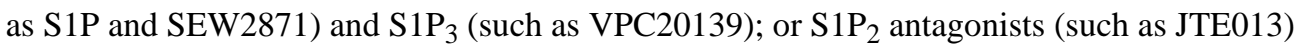
can also enhance the mobilization and homing processes. 